\title{
Prevalência e fatores de risco para a retinopatia da prematuridade: estudo com 450 pré-termos de muito baixo peso
}

\author{
Prevalence and risk factors for retinopathy of prematurity: \\ study with 450 very low birth weight preterm infants
}

João Borges Fortes Filho', Gabriela Unchalo Eckert ${ }^{2}$, Fabiana Borba Valiatti ${ }^{3}$, Marlene Coelho da Costa ${ }^{4}$, Pedro Paulo Bonomo ${ }^{5}$, Renato Soibelmann Procianoy ${ }^{6}$

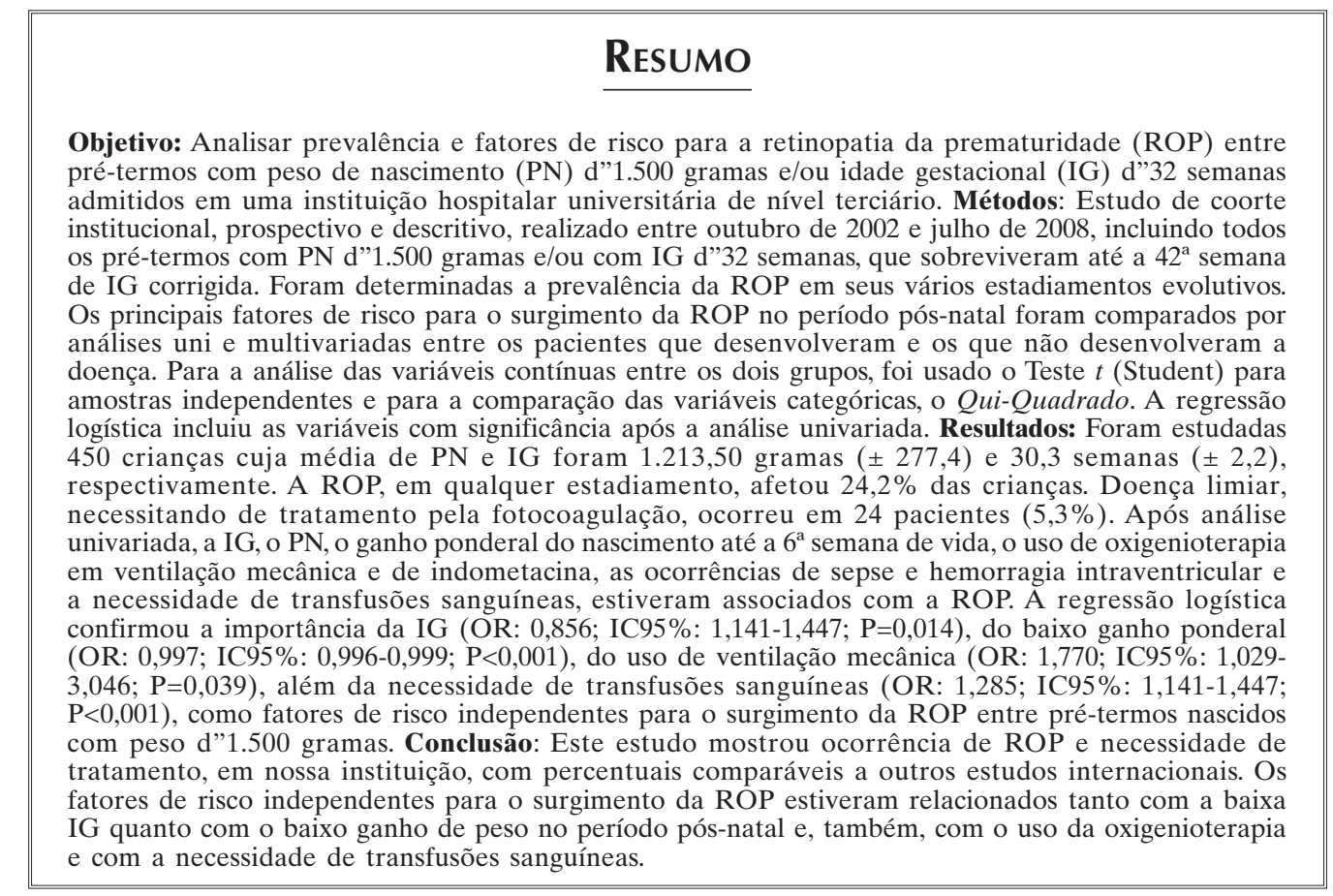

Descritores: Retinopatia da prematuridade/prevenção e controle; Prematuro; Recém-nascido de muito baixo peso; Fatores de risco; Prevalência

\footnotetext{
'Professor de Oftalmologia da Faculdade de Medicina da Universidade Federal do Rio Grande do Sul - UFRGS - Porto Alegre (RS), Brasil; Pós-graduando (Doutorado) em Oftalmologia pela Universidade Federal de São Paulo (UNIFESP), São Paulo (SP), Brasil; Pesquisador do PROROP - Programa de Prevenção da Cegueira pela Retinopatia da Prematuridade no Hospital de Clínicas de Porto Alegre - HCPA - Porto Alegre (RS), Brasil;

2Pesquisadora do PROROP - Programa de Prevenção da Cegueira pela Retinopatia da Prematuridade no Hospital de Clínicas de Porto Alegre - HCPA - Porto Alegre (RS), Brasil; Pós-graduanda (Doutorado) em Oftalmologia pela Universidade Federal de São Paulo UNIFESP - São Paulo, SP, Brasil;

${ }^{3}$ Pesquisadora do PROROP - Programa de Prevenção da Cegueira pela Retinopatia da Prematuridade no Hospital de Clínicas de Porto Alegre - HCPA - Porto Alegre (RS), Brasil;

Pós-graduanda (Mestrado) pela Universidade Federal do Rio Grande do Sul - UFRGS - Porto Alegre, RS, Brasil;

Enfermeira, pesquisadora do PROROP - Programa de Prevenção da Cegueira pela Retinopatia da Prematuridade no Hospital de Clínicas de Porto ${ }_{5}$ Alegre - HCPA - Porto Alegre (RS); Pós-graduanda pela Universidade Federal do Rio Grande do Sul - UFRGS - Porto Alegre (RS), Brasil;

${ }_{6}^{5}$ Doutor, Professor Adjunto da Oftalmologia da Universidade Federal de São Paulo - UNIFESP - São Paulo (SP), Brasil;

Professor Titular de Neonatologia da Faculdade de Medicina da Universidade Federal do Rio Grande do Sul Sul - UFRGS - Porto Alegre RS, Brasil; Pesquisador do PROROP - Programa de Prevenção da Cegueira pela Retinopatia da Prematuridade no Hospital de Clínicas de Porto Alegre - HCPA- Porto Alegre (RS), Brasil.
}

Recebido para publicação em: 4/8/2008 - Aceito para publicação em 9/1/2009 


\section{INTRODUÇÃO}

A retinopatia da prematuridade (ROP, do inglês retinopathy of prematurity) é uma entidade em permanente estudo em todo o mundo, devido ao aumento dos índices de sobrevivência entre crianças nascidas pré-termo de muito baixo peso (PMBP: nascidos com peso d"1.500 gramas). A ROP tornou-se uma das maiores causas de cegueira infantil (definida pela Organização Mundial de Saúde como cegueira ocorrendo até os 15 anos de idade) nos países desenvolvidos. A evolução natural da doença gera um elevado custo social e financeiro para toda a comunidade, por causar, em suas fases mais avançadas, dano severo e irreversível à visão, prejudicando, também, todo o desenvolvimento cognitivo e psicomotor da criança afetada $^{(1,2)}$. Nos anos mais recentes, devido à maior disponibilidade de hospitais habilitados ao atendimento de gestantes de risco e pela melhora na qualidade assistencial, aliada ao grande desenvolvimento tecnológico da Neonatologia, houve um aumento, também, na sobrevivência de prematuros de extremo baixo peso (PEBP: nascidos com peso d" 1.000 gramas) de $8 \%$ para mais de $35 \%$ na maioria dos países em desenvolvimento e, desde os anos 80, essa entidade tem sido considerada a maior causadora de cegueira infantil possível de ser prevenida, também nos países de economia ascendente, dentre os quais, encontra-se o Brasil ${ }^{(3-6)}$.

O I Workshop de Retinopatia da Prematuridade, realizado no Rio de Janeiro em outubro de 2002, organizado pelo Conselho Brasileiro de Oftalmologia, Sociedade Brasileira de Pediatria e Sociedade Brasileira de Oftalmologia Pediátrica, estimou que pudessem ocorrer de 500 a 1.500 casos de cegueira ou de deficiência visual severa, em decorrência da ROP, a cada ano, em nosso país. Neste encontro, ficou definido como critérios para a busca da doença o exame oftalmológico em todos os pré-termos com peso de nascimento (PN) d"1.500 gramas ou com idade gestacional (IG) d"32 semanas. As diretrizes, publicadas em $2007^{(7)}$, incluíram, também, nas triagens, todos os bebês nascidos com mais de 1.500 gramas, porém que desenvolveram outras co-morbidades relacionadas com o nascimento prematuro, tais como: sepse, hemorragia intraventricular, displasia bronco-pulmonar, enterocolite, meningite, entre outras, conforme parecer do neonatologista.

Existem estudos prévios ${ }^{(8.9)}$ que analisaram, de modo institucional e com número expressivo de pacientes incluídos, a prevalência da ROP em nosso país, porém todos foram publicados antes da divulgação das diretrizes brasileiras. Esse estudo teve como objetivo ana- lisar, prospectivamente, a prevalência da $\mathrm{ROP}$ e os possíveis fatores de risco (FR) para o surgimento da doença entre PMBP de uma unidade de neonatologia na Região Sul do Brasil, cujos pacientes foram avaliados de acordo com as diretrizes nacionais emitidas, em 2002, após o I Workshop ROP.

\section{Métodos}

Estudo de coorte, institucional, prospectivo e descritivo, incluindo todas as crianças nascidas pré-termo e admitidas na Unidade de Terapia Intensiva Neonatal (UTIN) de um hospital universitário de nível terciário, no período entre outubro de 2002 e julho de 2008 , que sobreviveram do momento do exame oftalmológico inicial, realizado entre a $4^{\mathrm{a}}$ e a $6^{\mathrm{a}}$ semana de vida, até completarem a $42^{\mathrm{a}}$ semana de idade pós-concepcional. Não houve critério de exclusão.

$\mathrm{O}$ exame oftalmológico em todos os pacientes consistiu de oftalmoscopia binocular indireta realizada, conforme anteriormente descrito ${ }^{(10)}$, após dilatação das pupilas com a associação dos colírios tropicamida $0,5 \%$ e fenilefrina $2,5 \%$, com uso de lente de 28 dioptrias (Nikon ${ }^{\circledast}$, Melville, NY, EUA) e com blefarostato para recém-nascidos (Alfonso Eye Speculum, Storz ${ }^{\oplus}$, Bausch \& Lomb Inc., San Dimas, CA, EUA). Depressão escleral foi utilizada, quando necessário, para melhor se identificar as alterações retinianas periféricas na Zona III.

As avaliações iniciaram entre a $4^{\mathrm{a}}$ e a $6^{\mathrm{a}}$ semana de vida e foram repetidas, periodicamente, de acordo com as diretrizes brasileiras ${ }^{(7)}$. Todos os pacientes foram examinados inicialmente na UTIN e, após a alta, foram seguidos ambulatorialmente até o momento de completada a vascularização retiniana na periferia temporal, ao redor da $42^{\mathrm{a}}$ semana de IG corrigida, ou até a completa estabilização da retinopatia nos pacientes que necessitaram de tratamento. Todos os exames oftalmológicos foram realizados pelos mesmos autores (JBFF, GUE e FBV).

O principal desfecho clínico foi o surgimento da ROP em qualquer estadiamento evolutivo. Os estadiamentos da doença foram registrados de acordo com a Classificação Internacional da ROP de 1984/1987 ${ }^{(11,12)}$ e corresponderam, sempre, ao pior grau de retinopatia encontrado em quaisquer dos olhos durante o seguimento dos pacientes. ROP severa ficou definida como doença em estadiamentos 3, doença limiar, 4 e 5 . Doença limiar foi definida de acordo com o Multicenter trial of cryotherapy for retinopathy of prematurity como o momento a partir do qual o risco de um desfecho anatômico ou funcional desfavorável, ou de progressão para ceguei- 
ra, ocorre em $50 \%$ dos pacientes ${ }^{(13)}$.

De acordo com o desfecho clínico, os prematuros foram divididos em dois grupos: Grupo sem ROP e Grupo com ROP. Foram considerados os seguintes FR: PN, IG (avaliada pela história, por ecografia obstétrica precoce e por exame clínico do pré-termo; método Ballard), ser adequado ou pequeno para a respectiva IG $(<10$ percentil), ganho ponderal do nascimento até a $6^{\mathrm{a}}$ semana de vida (definido como o peso na $6^{\mathrm{a}}$ semana de vida menos o PN), sexo, gemelaridade (nascido de gestação única ou múltipla), uso de oxigenioterapia em ventilação mecânica ou por nasal Continuous Positive Airway Pressure (nasal CPAP), uso de indometacina, surfactante e eritropoetina, escores de Apgar no $5^{\circ}$ minuto, desenvolvimento de sepse, meningite e qualquer grau de hemorragia intraventricular e a necessidade de transfusões sanguíneas. $\mathrm{O}$ diagnóstico para sepse foi clínico e por hemocultura, para meningite foi por cultura microbiológica e para hemorragia intraventricular, por ultrassonografia craniana. Não foi possível determinar a duração do uso de oxigênio. Essa variável foi considerada dicotômica (uso ou não uso de oxigenioterapia). Para a análise das variáveis contínuas entre os dois grupos, foi usado o Teste $t$ (Student) para amostras independentes e para a comparação das variáveis categóricas, o Qui-Quadrado.As variáveis consideradas para a regressão logística foram selecionadas por sua significância, após análise univariada. Os resultados foram significativos para $\mathrm{P}<0,05$. Todas as análises estatísticas foram realizadas com o programa SPSS (SPSS ${ }^{\circledR} 13.0$ for Windows ${ }^{\circledR}$. SPSS Inc., Chicago, IL, EUA). O protocolo do estudo foi aprovado pelo Comitê de Ética em Pesquisa da instituição sob o n ${ }^{\circ}$ 03-248, em 20/8/2003.

\section{Resultados}

De outubro de 2002 até julho de 2008, foram triados para a ROP e incluídos no estudo um total de 450 PMBP.As características gerais da população estudada podem ser vistas na Tabela 1 . Duzentas e cinqüenta e cinco crianças eram do sexo feminino (56,7\%), e 254 delas, nascidas com peso apropriado para sua respectiva IG. A média do PN e da IG entre todos os pré-termos da coorte foi $1.213,50$ gramas $( \pm 277,4)$ e 30,3 semanas $( \pm$ 2,2), respectivamente.

A prevalência da ROP, em seus vários estadiamentos evolutivos, foi $24,2 \%$. A prevalência por estadiamento pode ser vista na Tabela 2. ROP severa e doença limiar, necessitando tratamento, afetou 24 crianças $(5,3 \%)$. Dessas, 22 atingiram o estadiamento de ROP
Tabela 1

Características gerais da coorte de 450 prematuros de muito baixo peso

\begin{tabular}{lc}
\hline & n (\%) \\
\hline Sexo feminino / masculino & $255(56,7) / 195(43,3)$ \\
PIG $(<10$ percentil) & $196(43,6)$ \\
PN * em gramas & $1.213,50(277,4)$ \\
IG * em semanas & $30,3(2,2)$ \\
\hline
\end{tabular}

n: número de pacientes; \%: percentual ; PIG: pequeno para a idade gestacional; PN: peso de nascimento; IG: idade gestacional;

* dados apresentados com média (desvio padrão)

Tabela 2

\section{Prevalência de retinopatia da prematuridade entre $\mathbf{4 5 0}$ prematuros de muito baixo peso no período entre 2002 e 2008}

\begin{tabular}{llr}
\hline ROP & & $\mathbf{n ~ ( \% )}$ \\
\hline Sem ROP & & $341(75,8)$ \\
Com ROP & & $109(24,2)$ \\
& ROP 1 & $48(10,7)$ \\
& ROP 2 & $37(8,2)$ \\
& ROP 3 & $22(4,9)$ \\
& ROP 4 & $1(0.2)$ \\
Total & ROP 5 & $1(0,2)$ \\
\hline
\end{tabular}

ROP: retinopatia da prematuridade; *: ROP severa (estadiamentos $3,4,5$ ); n: número de pacientes; \%: percentual de pacientes

* dados apresentados com média (desvio padrão)

3, doença limiar. Apenas duas crianças desenvolveram ROP em estadiamentos 4 e 5 (uma em cada estadiamento; $0,2 \%$ cada). A Tabela 3 ilustra a distribuição da ocorrência dos estadiamentos da ROP por faixas de peso de nascimento.

Na Tabela 4, estão apresentados os principais FR relacionados com o surgimento da ROP após análise univariada. O PN, a IG, o baixo ganho ponderal do nascimento até a $6^{\mathrm{a}}$ semana de vida foram significativamente menores nos pacientes que desenvolveram ROP, enquanto o uso de oxigenioterapia em ventilação mecânica, o uso de indometacina, a ocorrência de sepse e de hemorragia intraventricular em qualquer estadiamento, além da necessidade de transfusões sanguíneas foram significativamente mais incidentes entre os pacientes que desenvolveram a ROP.

Depois de ajustes por regressão logística (Tabela 5), a menor IG, o baixo ganho ponderal, o uso de ventilação mecânica e a necessidade de transfusões sanguíneas foram considerados FR independentes para o surgimento da ROP nesta coorte de 450 pacientes PMBP, 
Tabela 3

Estadiamentos da retinopatia da prematuridade por faixas de peso de nascimento

\begin{tabular}{lccccccc}
\hline \multicolumn{1}{c}{ PN } & Sem ROP & ROP 1 & ROP 2 & ROP 3 & ROP 4 & ROP 5 & Total \\
\hline$\leq 500$ gramas & 0 & 0 & 0 & 0 & 0 & 0 & 0 \\
501 - 750 gramas & 11 & 1 & 9 & 6 & 0 & 1 & 28 \\
751 - 1.000 gramas & 45 & 11 & 10 & 10 & 1 & 0 & 77 \\
1001 - 1.500 gramas & 225 & 35 & 18 & 6 & 0 & 0 & 284 \\
$>$ 1.500 gramas & 60 & 1 & 0 & 0 & 0 & 0 & 61 \\
Total de pacientes & 341 & 48 & 37 & 22 & 1 & 1 & 450 \\
\hline
\end{tabular}

ROP: Retinopatia da prematuridade; $\mathrm{PN}$ : peso de nascimento

Tabela 4

Análise univariada dos fatores de risco para o surgimento da retinopatia da prematuridade em toda a coorte

\begin{tabular}{lccc}
\hline \multicolumn{1}{c}{ Variáveis } & $\begin{array}{c}\text { Grupo sem ROP } \\
\mathbf{3 4 1} \text { pacientes }\end{array}$ & $\begin{array}{c}\text { Grupo com ROP } \\
\mathbf{1 0 9} \text { pacientes }\end{array}$ & Valor de p \\
\hline Peso de nascimento em gramas * & $1.262,5 \pm 259,9$ & $1.059,9 \pm 275,5$ & $<0,001$ \\
Idade gestacional em semanas * & $30,7 \pm 2,1$ & $29,2 \pm 2,3$ & $<0,001$ \\
PIG (<10\% percentil) & $69( \pm 27,2)$ & $40( \pm 20,4)$ & 0,120 \\
Ganho ponderal * & $662,3 \pm 260,3$ & $468,4 \pm 255,7$ & $<0.001$ \\
Índice de Apgar no 5 minuto * & $7,9 \pm 1,8$ & $7,7 \pm 1,6$ & 0,571 \\
Uso de oxigênio em ventilação mecânica & $151(44,3 \%)$ & $67(61,5 \%)$ & 0,002 \\
Uso de oxigênio em CPAP nasal & $262(76,8 \%)$ & $85(78,0 \%)$ & 0,896 \\
Uso de eritropoetina & $257(76,0 \%)$ & $86(79,6 \%)$ & 0,512 \\
Usp de indometacina & $93(27,3 \%)$ & $45(41,3 \%)$ & 0,008 \\
Uso de surfactante & $149(43,7 \%)$ & $57(52,3 \%)$ & 0,123 \\
Necessidade de transfusões sanguíneas & $159(46,6 \%)$ & $88(80,7 \%)$ & $<0,001$ \\
Presença de hemorragia intraventricular & $52(15,2 \%)$ & $26(23,9 \%)$ & 0,043 \\
Presença do ducto arterial & $39(11,4 \%)$ & $18(16,5 \%)$ & 0,186 \\
Ocorrencia de sepse & $213(62,5 \%)$ & $82(75,2 \%)$ & 0,015 \\
Meningite & $24(7,0 \%)$ & $6(5,5 \%)$ & 0,665 \\
Gemelaridade & $55(16,1 \%)$ & $20(18,3 \%)$ & 0,658 \\
Sexo feminino & $190(55,7 \%)$ & $65(59,6 \%)$ & 0,506 \\
\hline
\end{tabular}

ROP: retinopatia da prematuridade; CPAP nasal:nasal Continuous Positive Airway Pressure; PIG: pequeno para a idade gestacional; * Dados apresentados com média \pm desvio padrão

Tabela 5

Razão de chances (Odds ratio) para o surgimento da retinopatia da prematuridade após regressão logística ajustada

\begin{tabular}{lccr}
\hline & OR & $\begin{array}{c}\text { Intervalo de } \\
\text { Confiança 95\% }\end{array}$ & $\begin{array}{r}\text { Valor } \\
\text { de p p }\end{array}$ \\
\hline Idade gestacional & 0,856 & $0,756-0,968$ & 0,014 \\
Ganho ponderal & 0,997 & $0,996-0,999$ & $<0,001$ \\
Uso de oxigenioterapia em VM & 1,770 & $1,029-3,046$ & 0,039 \\
Necessidade de transfusões & 1,285 & $1,141-1,447$ & $<0,001$ \\
\hline
\end{tabular}

ROP: retinopatia da prematuridade; VM: ventilação mecânica 
que foram triados e acompanhados evolutivamente de acordo com os critérios definidos nas diretrizes brasileiras para a detecção da ROP.

\section{DiscussÃo}

A ROP é uma causa importante de cegueira em países com baixa mortalidade infantil. Gilbert et al. ${ }^{(2)}$ relataram que a ROP é predominante em pré-termos nascidos com $\mathrm{PN}<1.000$ gramas nos países industrializados e está emergindo como uma causa importante de cegueira infantil também nos países de economia ascendente, como o Brasil e em outros da América Latina, bem como na Ásia e no leste europeu em função da maior sobrevivência de PMBP.

Estudos sugerem que a incidência e severidade da ROP mostram um relacionamento inverso com o PN e com a IG ${ }^{(14,15)}$, com poucos casos da ROP severa sendo diagnosticados em bebês nascidos com mais de 1.500 gramas ou com IG maior do que 32 semanas. A ROP severa tem maior incidência entre crianças nascidas com IG $<28$ semanas ou com PN $<1.000$ gramas, embora ainda sejam relatados casos de ROP severa em bebês nascidos com IG entre 34-35 semanas e PN >1.500 gramas, principalmente, na presença de muitas co-morbidades relacionadas com o nascimento pré-termo, como o relatado na Índia, China e na Lituânia ${ }^{(16-18)}$.

Nossa instituição é um hospital universitário de nível terciário, situado em uma região metropolitana, abrangendo uma população em torno de 4 milhões de habitantes. Sua UTIN, considerada de referência para gestantes de alto risco no sul do Brasil, tem 20 leitos para terapia intensiva com possibilidade de ventilação mecânica e com oximetria de pulso podendo ser utilizada em todos os pacientes. Durante o período considerado para o estudo, foram submetidos à triagem para a detecção da ROP um total de 450 pré-termos correspondendo a $92.6 \%$ de todos os nascimentos no grupo de risco, de acordo com os critérios definidos para o Brasil, após o ano de $2002^{(7)}$.

No Brasil, Graziano et al ${ }^{(8)}$ encontraram $29,1 \%$ de ROP em qualquer estadiamento entre 102 bebês prematuros com PN d"1.500 gramas. Esse estudo chamou a atenção para a alta prevalência de ROP entre pré-termos com PN d"1.000 gramas (78,5\%) e com IG d"30 semanas $(72,7 \%)$. Em outro estudo, Moraes et al. ${ }^{(9)}$ mostraram a ocorrência de $90 \%$ de ROP em bebês com PN d"1.000 gramas. Embora esses importantes estudos brasileiros, baseados em dados de unidades neonatais isoladas, sejam da década passada e, portanto, anteriores à publicação das diretrizes brasileiras para a detecção da ROP, a prevalência da doença em nossa instituição foi inferior ao relatado por aqueles autores. Em estudo anteriormente publicado ${ }^{(19)}$, verificamos a prevalência de $48,9 \%$ de ROP em qualquer estadiamento, quando analisamos apenas os pré-termos com $\mathrm{PN}<1.000$ gramas, e que essa prevalência fica reduzida a apenas $18,2 \%$ de ROP em qualquer estadiamento, se considerarmos somente os pacientes com PN >1.000 gramas. Estudo recente, publicado por Bonotto et al. ${ }^{(20)}$, em 2007, mostrou dados sobre 286 prematuros atendidos no período entre 1992 e 1999, na cidade de Joinville em Santa Catarina. Esse interessante trabalho, que incluiu pré-termos nascidos com IG d"37 semanas, mostrou uma prevalência geral da ROP em $20 \%$ e que os prematuros que necessitaram de tratamento (ao redor de $4 \%$ ) tinham menos do que 1.399 gramas e 33 semanas de IG ao nascimento. O estudo, por ter sido conduzido num período anterior ao da realização do I Workshop ROP, em 2002, incluiu prétermos com PN d"1.600 gramas ou com IG d" 37 semanas. Os autores relataram os principais $\mathrm{FR}$ associados ao surgimento da ROP, naquela coorte, mas não apresentaram regressão logística, para demonstrar maior nexo de causa e efeito dos mesmos sobre o surgimento da ROP.

Um estudo de base populacional, realizado por Hiraoka et al. ${ }^{(21)}$, no Japão, e publicado em 2002, relatou a sobrevivência de $85,6 \%$ de bebês com IG média de 26,74 semanas e PN médio de 782,25 gramas, uma sobrevivência muito mais alta do que tem sido possível de ser encontrada no Brasil nos dias de hoje. Os autores mencionam que $86,1 \%$ desses bebês desenvolveram ROP e $41 \%$ deles precisaram de tratamento por laser, igualmente uma frequência muito mais alta de ROP necessitando de tratamento do que observamos nos pacientes de nossa coorte. No relato japonês, 6 pacientes $(4,9 \%)$ desenvolveram descolamento da retina (estadiamentos 4 e 5). A necessidade de tratamento e a evolução para descolamento de retina no trabalho japonês estiveram muito acima do observado em nossa coorte de pacientes. Uma possível explicação para essas diferenças apontadas poderia ser o fato de que nossa coorte de pacientes tinha uma média de PN e de IG de 1.213,50 gramas $( \pm 277,4)$ e 30,3 semanas $( \pm 2,2)$, respectivamente, portanto bebês de maior PN e com maior IG do que no estudo realizado no Japão e, eventualmente, nossos pacientes poderiam ser menos propensos a desenvolver ROP, especialmente em suas formas mais graves, por serem maiores e mais maduros.

Markestad et al. ${ }^{(22)}$, na Noruega, relataram 59\% de sobrevivência entre PEBP, com índices variando des- 
de nenhuma sobrevivência entre bebês com IG d"23 semanas até $90 \%$ de sobrevivência entre nascidos com mais de 27 semanas. O mesmo estudo não mostrou ROP severa entre bebês nascidos com mais de 25 semanas de GA. Os autores noruegueses relataram incidência de ROP em estadiamento 3 em apenas 2,9\% dos pacientes. Em nossa coorte registramos apenas 1 paciente com estadiamento ROP 4, nascido com 30 semanas de IG e 1 paciente com estadiamento ROP 5, nascido com 29 semanas de IG. Nossa incidência de ROP 3, doença limiar, considerando todos os PMBP nascidos no período foi $5,3 \%$, quase duas vezes mais do que o relatado para a Noruega.

Nos EUA, Hussain et al. ${ }^{(23)}$, entre 1989 e 1997, analisaram, retrospectivamente, dados sobre 950 bebês, mostrando que $21,3 \%$ apresentaram ROP, mas somente 4,6\% desenvolveram ROP estadiamento 3 ou pior e nenhum dos pacientes nascidos com $\mathrm{PN}>1.000$ gramas ou IG > 28 semanas necessitaram de intervenção cirúrgica para a ROP. Esses dados americanos são similares aos observados em nossa instituição, onde 5,3\% de todos os bebês triados desenvolveram doença limiar e somente 6 pré-termos $(2,3 \%)$, com PN >1.000 gramas, necessitaram de tratamento por laser ${ }^{(24)}$.

Existem muitos trabalhos analisando FR de risco perinatal para a ROP. Patz et al. ${ }^{(25)}$, num estudo clínico controlado, demonstraram claramente a relação de causa e efeito entre a oxigenioterapia e o surgimento da ROP. A hipóxia e a hiperóxia, além das flutuações da tensão arterial do oxigênio, têm sido implicadas como fatores etiológicos para a ROP. Em nossa instituição, o controle sobre a oxigenioterapia segue parâmetros rígidos, com os pré-termos sendo permanentemente controlados, tanto em seus aspectos clínicos, quanto monitorados por oximetria de pulso com padrões de pressão de saturação entre 88-94\%. O corpo de enfermagem é periodicamente instruído sobre a relação entre o controle eficiente do oxigênio e o surgimento da ROP e a necessidade de se evitar flutuações, pois é possível prevenir o surgimento da ROP severa, na maioria dos casos ${ }^{(26)}$.

A ROP, no momento atual do conhecimento, é considerada uma doença multifatorial, sendo o uso do oxigênio apenas um dos seus fatores causadores. Em nosso estudo, os principais FR associados para o surgimento da ROP, após análise univariada, foram: a baixa IG, o baixo PN, o baixo ganho ponderal no período pós-natal, a necessidade do uso de oxigenioterapia em ventilação mecânica, o uso de indometacina, a ocorrência de sepse e de hemorragia intraventricular e a necessidade de transfusões sanguíneas.
Trabalhos que analisam múltiplos FR na ROP têm enfatizado a necessidade de se examinar, com cautela, os resultados das análises estatísticas, em função de que a ROP é uma doença multifatorial, que ocorre em pacientes deprimidos e bastante doentes e que tem um período de manifestação muito curto. Embora a análise multivariada seja o método de preferência para esses estudos, seus resultados podem ser instáveis em muitas ocasiões pela influência de uma variável sobre as outras (27). O PN, a IG, o uso do oxigênio e de drogas terapêuticas, como o surfactante e a indometacina, por seu aparecimento direto na maioria dos estudos envolvendo prematuros, pode ocultar a importância de outros fatores clínicos também participantes no desenvolvimento de formas severas da ROP como, por exemplo: sepse, doença da membrana hialina, asfixia perinatal, hemorragia intracraniana, presença de enterocolite necrotisante, entre outras ${ }^{(23,24)}$.

Muitos estudos demonstram relação significativa do PN e da IG como fatores independentes para o desenvolvimento da ROP. Essa situação poderia vir a ser melhor entendida se os pacientes de uma determinada coorte pudessem ser agrupados de acordo com diferentes faixas de PN e de IG, desde que se tivesse uma quantidade suficiente de desfechos em cada grupo (surgimento da ROP, ou da ROP severa), caso contrário, os resultados poderiam ser confundidos por chance estatística, uma vez que todos os pacientes incluídos nos estudos sobre a ROP já são pacientes que têm o PN e a IG mais baixos, quando comparados com uma população normal. A interferência de uma variável sobre as outras difere, em muito, dependendo dos modelos estatísticos utilizados para a regressão logística em cada estudo ${ }^{(28)}$.

A ROP afeta mais freqüentemente os pré-termos menores e os clinicamente mais debilitados, mas pode ocorrer, também, em pacientes de maior PN. Ainda não está suficientemente demonstrado se as formas mais severas da doença estão associadas com as intervenções terapêuticas necessárias para a manutenção da vida do paciente nascido pré-termo ou com a severidade das co-morbidades associadas com a prematuridade ${ }^{(29,30)}$. Nosso estudo demonstrou esse fato, ao verificarmos que as co-morbidades, ocorrência de sepse e hemorragia intraventricular e as intervenções terapêuticas, uso de oxigenioterapia em ventilação mecânica, uso de indometacina e necessidade de transfusões, foram significativamente maiores entre os pacientes que desenvolveram a ROP do que entre aqueles que não a desenvolveram.

A presença de hemorragia intracraniana em qualquer estadiamento evolutivo foi um FR para a ROP com 
significância em nosso estudo. A relação entre esta ocorrência e o surgimento da ROP também tem sido motivo de pesquisa. Procianoy et al. ${ }^{(31)}$, em 1981, relataram uma associação significativa entre ROP cicatricial e a ocorrência de hemorragia intracraniana ao estudarem 138 PMBP. Christiansen ${ }^{(32)}$,em 2002, relatou uma associação significativa entre o grau da hemorragia intracraniana e o estadiamento da ROP numa coorte de 60 PMBP, onde 17 desenvolveram hemorragia em estadiamento severo (graus III e IV) e 49, apenas hemorragias menos severas (graus 0 a II). Sessenta e quatro por cento dos RNP que desenvolveram graus severos de hemorragia intracraniana apresentaram estadiamentos de ROP 3 ou pior. No Brasil, Tavano et al ${ }^{(33)}$ publicaram um estudo, em 1996, onde relacionaram, significativamente, a ROP com hemorragia intracraniana, sugerindo uma etiologia vascular na patogênese de ambas as doenças, conforme observado anteriormente por Procianoy ${ }^{(31)}$.

Em estudo anterior, evidenciamos a importância da implementação da triagem neonatal para a busca da ROP em outras unidades de neonatologia no país e que o critério brasileiro para a detecção de ROP foi efetivo em descobrir todos os casos de doença limiar ocorridos na coorte ${ }^{(24)}$. Nosso estudo demonstrou que a triagem neonatal impediu a ocorrência de cegueira em 23 de nossos pacientes. É importante salientar que nossas observações estão baseadas em dados de uma única unidade de neonatologia, que não devem ser extrapoladas para pacientes nascidos ou cuidados em unidades com características ou com possibilidades de cuidados neonatais diferentes das praticadas num hospital de nível terciário. Nossos resultados podem ser comparados com estudos similares originados de países industrializados, onde existe excelência de cuidado perinatal ${ }^{(34)}$.É possível que a incidência de ROP venha a ser mais alta em nossa instituição, uma vez que não temos dados sobre bebês falecidos posteriormente à alta hospitalar. Esses pacientes, por terem saúde mais debilitada, poderiam ter alcançado estadiamentos maiores da ROP, porém não sobreviveram para essa constatação.

\section{Conclusão}

As médias do PN e da IG em nossa coorte de pacientes foram, respectivamente, 1.213,50 gramas $( \pm$ $277,4)$ e 30,3 semanas $( \pm 2,2)$. As prevalências da ROP em qualquer estadiamento e da ROP severa, necessitando de tratamento, em nosso estudo podem ser comparadas com trabalhos originados de países ou de instituições com elevada qualidade de atendimento perinatal. Os fatores de risco independentes para o surgimento da
ROP estiveram relacionados tanto com a baixa IG quanto com o baixo ganho de peso no período pós-natal e, também, com o uso da oxigenioterapia e com a necessidade de transfusões sangüíneas.

A implementação de programas de triagem neonatal para a detecção e tratamento precoce da ROP em outros hospitais no Brasil contribuiria, em muito, para a diminuição dessa doença, que é a maior causa de cegueira infantil evitável em nosso país.

\section{Abstract}

Purpose: This study aims to analyze prevalence and risk factors for retinopathy of prematurity (ROP) among preterms with birth weight $(B W) d " 1,500$ grams and/or gestational age $(G A)$ d" 32 weeks, admitted in an University level 3 institution. Methods: An institutional, prospective and descriptive cohort study including all preterms with BW d"1,500 grams and/or GA d" 32 weeks, who survived from birth to the $42^{\text {nd }}$ week of postmentrual age, was carried out from October 2002 to July 2008. The prevalence of any stage ROP and the main risk factors of postnatal period for ROP were compared by uni and multivariate analysis. Continuous variables were analysed by Student's $t$ - Test. Chi-square was used to compare cathegoric variables. To the logistic regression were included all of the significant variables after univariate analysis. Results: Retinopathy in any evolutive stage has affected $24.2 \%$ of the babies. Threshold disease needing treatment has appeared in 24 patients (5.3\%). After univariate analysis, $G A, B W$, use of mechanical ventilation and indomethacin, occurrence of intraventricular hemorrhage and sepsis, and need of blood transfusions, have been associated with ROP. Logistic regression has confirmed the great importance of GA (OR:0,856; IC95\%: 1,141-1,447; $P=0,014)$, low weight gain from birth to the $6^{\text {th }}$ week of life (OR: 0,997; IC95\%: 0,996-0,999; $P<0,001$ ), use of mechanical ventilation (OR: 1,770; IC95\%: 1,0293,046; $P=0,039)$, and need of blood transfusions (OR: 1,285; IC95\%: 1,141-1,447; $P<0,001$ ), as independent risk factors for developing of ROP among preterms with $B W$ d"1,500 grams. Conclusion: This study has shown prevalence of ROP and need of treatment among this institution's preterms comparable to other international studies. The main risk factors for ROP in this cohort were related with the $B W$, the postnatal low weight gain, and therapies like mechanical ventilation and blood transfusions.

Keywords: Retinopathy of prematurity/prevention and control; Premature, infant; Infant, very low birth weight; Risk factors; Prevalence 


\section{REFERÊNCIAS}

1. Wheatley CM, Dickinson JL, Mackey DA, Craig JE, Sale MM. Retinopathy of prematurity: recent advances in our understanding. Br J Ophthalmol. 2002;86(6):696-700. Review.

2. Gilbert C, Fielder A, Gordillo L, Quinn G, Semiglia R, Visintin $\mathrm{P}$, Zin A; International NO-ROP Group. Characteristics of infants with severe retinopathy of prematurity in countries with low, moderate, and high levels of development: implications for screening programs. Pediatrics. 2005;115(5):e518-25.

3. Foster A, Gilbert C. Epidemiology of childhood blindness. Eye. 1992;6(Pt 2):173-6.

4. Steinkuller PG, Du L, Gilbert C, Foster A, Collins ML, Coats DK. Childhood blindness. J AAPOS. 1999;3(1):26-32.

5. Gilbert C. Retinopathy of prematurity - the "second lull"? Br J Ophthalmol. 2001;85(9):1017-9.

6. Gilbert C, Foster A. Childhood blindness in the context of VISION 2020 - the right to sight. Bull World Health Organ. 2001;79(3):227-32.

7. Zin A, Florêncio T, Fortes Filho JB, Nakanami CR, Gianini N Graziano RM, Moraes N. Proposta de diretrizes brasileiras do exame e tratamento de retinopatia da prematuridade (ROP). Arq Bras Oftalmol. 2007;70(5):875-83.

8. Graziano RM, Leone CR, Cunha SL, Pinheiro AC. Prevalência da retinopatia da prematuridade em Recém-nascidos de muito baixo peso. J Pediatr (Rio J). 1997;73(6):377-82.

9. Moraes NSB, Bonomo PPO, Almeida MFB. Retinopatia da prematuridade: estudo prospectivo de 1342 casos. Arq Bras Oftalmol. 1995;58(4 Supl):255.

10. Fortes Filho JB. Retinopatia da prematuridade. Artigo de revisão. Rev Bras Oftalmol. 2006;65(4):246-58.

11. An international classification of retinopathy of prematurity. The Committee for the Classification of Retinopathy of Prematurity. Arch Ophthalmol. 1984;102(8):1130-4.

12. An international classification of retinopathy of prematurity. II. The classification of retinal detachment. The International Committee for the Classification of the Late Stages of Retinopathy of Prematurity. Arch Ophthalmol. 1987;105(7):90612. Erratum in: Arch Ophthalmol. 1987;105(11):1498

13. Multicenter trial of cryotherapy for retinopathy of prematurity. Preliminary results. Cryotherapy for Retinopathy of Prematurity Cooperative Group. Arch Ophthalmol. 1988;106(4):471-9.

14. Shah VA, Yeo CL, Ling YL, Ho LY. Incidence, risk factors of retinopathy of prematurity among very low birth weight infants in Singapore. Ann Acad Med Singapore. 2005;34(2):169-78.

15. Lermann VL, Fortes Filho JB, Procianoy RS. The prevalence of retinopathy of prematurity in very low birth weight newborn infants. J Pediatr (Rio J). 2006;82(1):27-32.

16. Sehgal A, Telang S, Passah SM, Jyothi MC. Maternal and neonatal profile and immediate outcome in ELBW babies. Indian Pediatr. 2003;40(10):991-5.

17. Fang PC, Kuo HK, Ko TY, Chen CC, Hwang KP, Chung MY Retinopathy of prematurity in larger preterm infants. Am J Perinatol. 2006;23(5):273-7.

18. Sirtautiene R, Bagdoniene R. Retinopathy of prematurity: is it time to change screening limits in Lithuania? Acta Med Lituanica. 2006;13(3):161-4.

19. Fortes Filho JB, Eckert GU, Procianoy L, Barros CK, Procianoy RS. Incidence and risk factors for retinopathy of prematurity in very low and in extremely low birth weight infants in a unit-based approach in southern Brazil. Eye. 2007 Jul 6. [Epub ahead of print]. doi:10.1038/sj.eye.6702924.
20. Bonotto LB, Moreira ATR, Carvalho DS. Prevalência de retinopatia da prematuridade em prematuros atendidos no período de 1992-1999 em Joinville (SC): avaliação de riscos associados - "screening". Arq Bras Oftalmol. 2007;70(1):55-61.

21. Hiraoka M, Watanabe T, Kawakami T, Ito R, Takigawa I, Suzumura H, et al. [Retinopathy of prematurity in extremely low birth weight infants: a Tokyo multicenter study]. Nippon Ganka Gakkai Zasshi. 2004;108(10):600-5. Japanese.

22. Markestad T, Kaaresen PI, Ronnestad A, Reigstad H, Lossius K, Medbo S, Zanussi G, Engelund IE, Skjaerven R, Irgens LM; Norwegian Extreme Prematurity Study Group. Early death, morbidity, and need of treatment among extremely premature infants. Pediatrics. 2005;115(5):1289-98.

23. Hussain N, Clive J, Bhandari V. Current incidence of retinopathy of prematurity, 1989-1997. Pediatrics. 1999;104(3):e26.

24. Fortes Filho JB, Barros CK, Costa MC, Procianoy RS. Resultados de um programa de prevenção da cegueira pela retinopatia da prematuridade na Região Sul do Brasil. J Pediatr (Rio J). 2007;83(3):209-16.

25. Patz A. Oxygen administration to the premature infant. A two-edged sword. Am J Ophthalmol. 1967;63(2):351-3.

26. Costa MC, Eckert GU, Valiatti FB, Bonomo PP, Fortes Filho JB. Incidência da retinopatia e a participação da enfermagem na prevenção da cegueira pela Retinopatia da Prematuridade no Hospital de Clínicas de Porto Alegre: estudo prospectivo observacional descritivo. Online Braz J Nurs [Internet]. 2007;6(3). Available from: http://www.uff.br/ objnursing/index.php/nursing/article/view/j.16764285.2007.976/257

27. Katz MH. Multivariable analysis: a primer for readers of medical research. Ann Intern Med. 2003;138(8):644-50.

28. Ikeda H, Kuriyama S. Risk factors for retinopathy of prematurity requiring photocoagulation. Jpn J Ophthalmol. 2004;48(1):68-71.

29. Italian multicentre study on retinopathy of prematurity. The Italian ROP Study Group. Eur J Pediatr. 1997;156(12):939-43.

30. Jalali S, Matalia J, Hussain A, Anand R. Modification of screening criteria for retinopathy of prematurity in India and other middle-income countries. Am J Ophthalmol. 2006;141(5):966-8.

31. Procianoy RS, Garcia-Prats JA, Hittner HM, Adams JM, Rudolph AJ. An association between retinopathy of prematurity and intraventricular hemorrhage in very low birth weight infants. Acta Paediatr Scand. 1981;70(4):473-7.

32. Christiansen SP, Fray KJ, Spencer T. Ocular outcomes in low birth weight premature infants with intraventricular hemorrhage. J Pediatr Ophthalmol Strabismus. 2002;39(3):157-65.

33. Tavano V, Nogueira R, Moraes NSB, Farah ME. Associação entre retinopatia da prematuridade e hemorragia intraventricular em recém-nascidos. Arq Bras Oftalmol. 1996;59(4):373.

34. Quinn GE. Retinopatia da prematuridade no Brasil: um problema emergente: [editorial]. J Pediatr (Rio J). 2007;83(3):191-3.

\section{ENDEREÇO PARA CORRESPONDÊNCIA: \\ Prof. Dr. João Borges Fortes Filho \\ Hospital de Clínicas de Porto Alegre \\ Rua Ramiro Barcelos, 2350 \\ CEP 90035-003 - Porto Alegre - RS - Brasil \\ E-mail: jbfortes@prorop.com.br}

